\title{
Spontaneous reossification of the sella in transsphenoidal reoperation associated with strontium ranelate
}

\author{
Maria Mercedes Pineyro', Daiana Arrestia', Mariana Elhordoy', Ramiro Lima², \\ Saul Wajskopf ${ }^{2}$, Raul Pisabarro' ${ }^{1}$ and Maria Pilar Serra' \\ 'Clínica de Endocrinología y Metabolismo and 2Neurocirugía, Hospital de Clínicas, Facultad de \\ Medicina, Universidad de la República, Montevideo, Uruguay
}

\author{
Correspondence \\ should be addressed \\ to M M Pineyro \\ Email \\ mercepin@gmail.com
}

\section{Summary}

Spontaneous reossification of the sellar floor after transsphenoidal surgery has been rarely reported. Strontium ranelate, a divalent strontium salt, has been shown to increase bone formation, increasing osteoblast activity. We describe an unusual case of a young patient with Cushing's disease who was treated with strontium ranelate for low bone mass who experienced spontaneous sellar reossification after transsphenoidal surgery. A 21-year-old male presented with Cushing's features. His past medical history included delayed puberty diagnosed at 16 years, treated with testosterone for 3 years without further work-up. He was diagnosed with Cushing's disease initially treated with transsphenoidal surgery, which was not curative. The patient did not come to follow-up visits for more than 1 year. He was prescribed strontium ranelate $2 \mathrm{~g}$ orally once daily for low bone mass by an outside endocrinologist, which he received for more than 1 year. Two years after first surgery he was reevaluated and persisted with active Cushing's disease. Magnetic resonance image revealed a left $4 \mathrm{~mm}$ hypointense mass, with sphenoid sinus occupation by a hyperintense material. At repeated transsphenoidal surgery, sellar bone had a very hard consistency; surgery was complicated and the patient died. Sellar reossification negatively impacted surgery outcomes in this patient. While this entity is possible after transsphenoidal surgery, it remains unclear whether strontium ranelate could have affected sellar ossification.

\section{Learning points:}

- Delayed puberty can be a manifestation of Cushing's syndrome. A complete history, physical examination and appropriate work-up should be performed before initiating any treatment.

- Sellar reossification should always be taken into account when considering repeated transsphenoidal surgery. Detailed preoperative evaluation of bony structures by computed tomography ought to be performed in all cases of reoperation.

- We speculate if strontium ranelate may have affected bone mineralization at the sellar floor. We strongly recommend that indications for prescribing this drug should be carefully followed.

\section{Background}

Spontaneous reossification of the sellar floor after transsphenoidal surgery has been rarely reported (1).

Bone regrowth or regeneration at the sellar floor may ensue after transsphenoidal surgery, particularly if reoperation is deferred for a significant period of time.
Strontium ranelate, a divalent strontium salt, has been shown to increase bone formation, increasing osteoblast activity (2). It is registered as a prescription drug for the treatment of postmenopausal osteoporosis in Europe and various other countries. 
It is currently indicated in males and postmenopausal females with severe osteoporosis, at high risk of fracture, who cannot be treated with other osteoporosis medicines and do not have contraindications for this drug. It is not FDA approved for use in USA.

Most common side effects include diarrhea, nausea and headaches. In addition, post-marketing surveillance reported some cases of a rare hypersensitivity syndrome (DRESS: Drug Reaction, Eosinophilia and Systemic Symptoms).

We present a case of Cushing's disease treated with strontium ranelate for low bone mass that presented with significant reossification of the sellar floor at reoperation.

\section{Case presentation}

A 21-year-old male was referred for evaluation of Cushing's syndrome. Past medical history included delayed puberty diagnosed at 16 years, treated with testosterone for 3 years without further work-up. Additionally, HTN was diagnosed at age 18 years, treated with indapamide: $1.5 \mathrm{mg} /$ day, metoprolol: $50 \mathrm{mg} /$ day and ramipril: $5 \mathrm{mg} /$ day. He also suffered vertebral fractures after a minor car accident.

He had gained weight over the last 5 years. He complained of easy bruising, muscle weakness and intermittent headaches. He denied use of exogenous glucocorticoids. Physical examination revealed normal weight (BMI 24), Cushingoid features with facial plethora, dorsocervical and supraclavicular fat deposition. In addition, thin skin with ecchymosis was noted. No abdominal striae were present. Also, the abdomen was prominent, with no organomegaly. Proximal myopathy was evident.

\section{Investigation}

Urinary free cortisol (UFC) was $578 \mu \mathrm{g} / 24 \mathrm{~h}$ (reference range: 28.4-213.7), serum cortisol level after an overnight $1 \mathrm{mg}$ dexamethasone suppression test was $25.8 \mu \mathrm{g} / \mathrm{dL}$ (reference range $<1.8$ ) and morning ACTH was $83 \mathrm{pg} /$ $\mathrm{mL}$ (7.2-63.3). Adrenocorticotropin hormone (ACTH)dependent Cushing's syndrome was diagnosed.

Brain magnetic resonance imaging (MRI) showed a left $5 \mathrm{~mm}$ hypointense lesion (Fig. 1).

An $8 \mathrm{mg}$ dexamethasone suppression test showed $82.4 \%$ serum cortisol suppression (from 33.23 to $5.67 \mu \mathrm{g} / \mathrm{dL}$ ). Bilateral inferior petrosal sinus sampling was not done because it is not available in the country. Anterior pituitary function was normal except for hypogonadotropic hypogonadism. Dual-energy X-ray

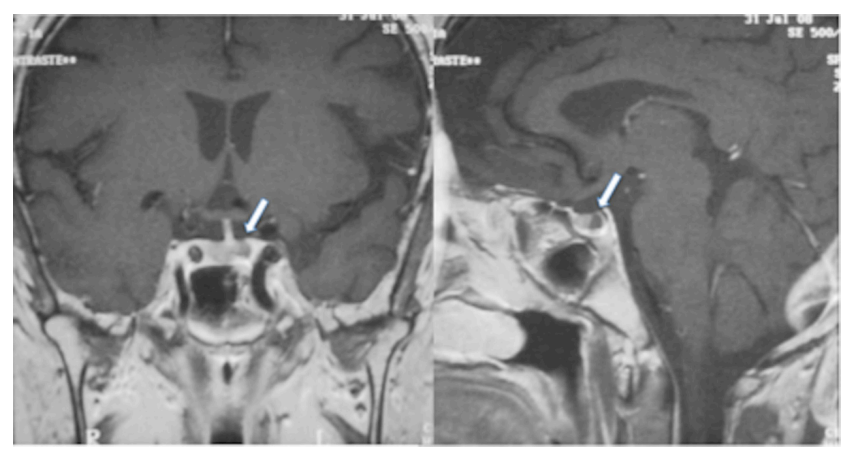

Figure 1

Coronal and sagittal post-contrast MRI at diagnosis showed a left $5 \mathrm{~mm}$ hypointense lesion.

absorptiometry showed low bone mineral density on lumbar spine ( $Z$-score: -4.4 ) and femoral head ( $Z$-score: -3.8). He had normal serum calcium, serum phosphate, renal and hepatic function, complete blood count, a 24-h urinary calcium excretion and 25-hydroxyvitamin D $[25(\mathrm{OH}) \mathrm{D}]$. Secondary low bone mass due to endogenous hypercortisolism and hypogonadism was diagnosed.

\section{Treatment}

Low bone mass was treated with calcium, vitamin D $90000 \mathrm{U}$ every 3 months and pamidronate $90 \mathrm{mg}$ IV every 3 months (3 times).

He did not receive any medical treatment for hypercortisolism before surgery.

The patient underwent transnasal transeptal microscopic transsphenoidal surgery without complications, and an adenoma was excised.

\section{Outcome and follow-up}

Postoperative morning cortisol levels remained high $(24.2 \mu \mathrm{g} / \mathrm{dL})$. The patient did not receive any further medical treatment after surgery, as he did not come to follow-up visits for more than 1 year. He was prescribed strontium ranelate $2 \mathrm{~g}$ orally once daily by an outside endocrinologist, which he received for more than 1 year.

Almost 2 years after the first surgery, he was reevaluated and persisted with active Cushing's disease. At this time, UFC was $1413 \mu \mathrm{g} / \mathrm{dL}$ (28.4-213.7). MRI revealed a left $4 \mathrm{~mm}$ hypointense mass, with sphenoid sinus occupation by a hyperintense material (Fig. 2).

Computed tomography (CT) scan showed sellar reossification (Fig. 3). At repeated transnasal transeptal microscopic transsphenoidal surgery sellar bone had a very hard consistency; surgery was complicated 


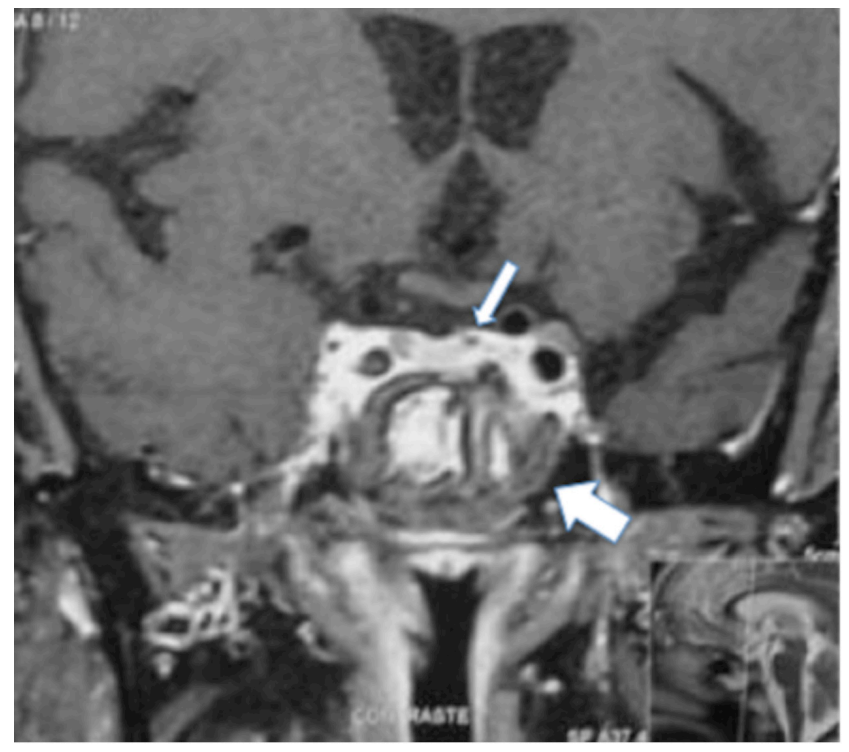

Figure 2

Coronal post-contrast MRI showed a left $4 \mathrm{~mm}$ hypointense mass (thin arrow), with sphenoid sinus occupation by a hyperintense material (block arrow)

with severe hemorrhage and the patient died septic 4 days later.

\section{Discussion}

This case is remarkable for a spontaneous reossification of the sellar floor in a patient operated for an ACTH-secreting pituitary tumor, which lead to a complicated surgery.

Pubertal disorders due to hypogonadotropic hypogonadism induced by hypercortisolism may be a

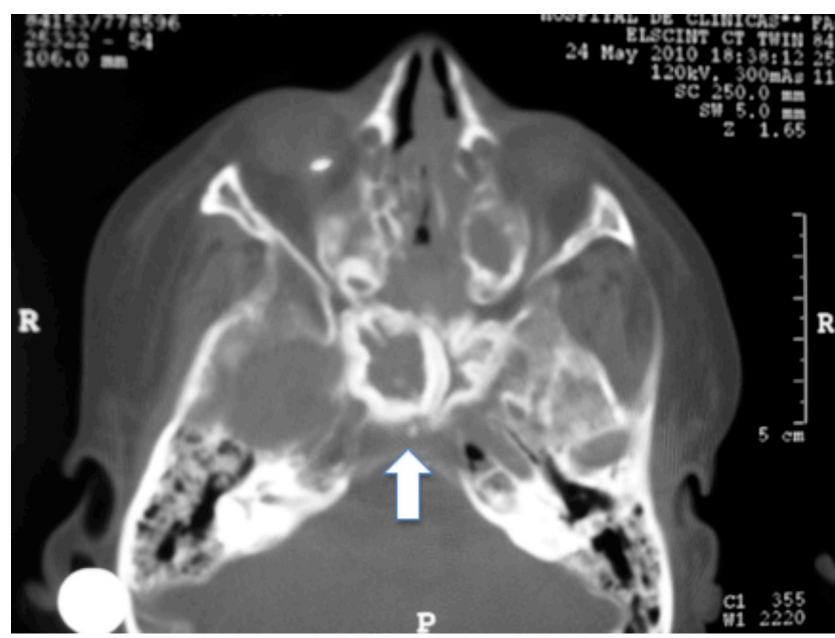

Figure 3

CT scan (bone window) previous to second transsphenoidal surgery showed sellar reossification. presentation of Cushing's syndrome. A complete history and physical examination, as well as appropriate work-up at that time may have led to early diagnosis and treatment.

There have been few cases of spontaneous sellar reossification after reoperation reported in the literature, more frequently in GH-secreting adenomas $(1,3)$.

Zona and coworkers (3) reported two cases (one GH-secreting adenoma) of diffuse and very hard reossification with reconstruction of the sellar floor and sphenoid wall after a few months of transsphenoidal surgery. In one of the cases, there was a complete ossification of the sinus cavity. In both cases, at the initial transsphenoidal surgery, the sella and sphenoid were left open in view of a second operation.

Recently Yahia-Cherif and coworkers (4) reported bony regeneration of the sella after transsphenoidal surgery in 16 of 17 patients with macroadenomas. The reduction in the size of the sellar floor opening was evaluated comparing cranial CT scans, measuring the maximum opening diameter in the transverse and sagittal planes. Overall, there was a significant median reduction of the bony opening from $8.8 \mathrm{~mm}$ to $4.2 \mathrm{~mm}$ in the transverse plane and from 4.8 to $2.9 \mathrm{~mm}$ in the sagittal plane. These changes where seen in a median time of 36 months. In 3 patients, an important ossification of the sphenoid sinus was present. In the only patient with ACTH-secreting macroadenoma, a $1.2 \mathrm{~mm}$ regeneration at the transverse plane was noted, but nothing was recorded in the sagittal plane.

Strontium ranelate is a drug for the treatment of osteoporosis that has dual mechanism of action on bone metabolism. It has been shown to decrease bone reabsorption by osteoclasts and to favor bone formation by osteoblast (2).

Moreover, there is evidence that strontium ranelate accumulates in bone tissue. It has been shown that is mainly deposited in young bone (5). This drug is incorporated into the callus of fractures in rats (6). In addition, it has a positive effect in bone fracture healing in osteoporotic rat models.

In humans, strontium ranelate had a positive effect on 3D microarchitecture in bone biopsies of osteoporotic postmenopausal women (7). Tarantino and coworkers (8) reported two cases in which treatment with strontium ranelate, calcium and vitamin D3 led to clinical improvement as well as fracture healing with increased callus formation. Furthermore, Aslam and coworkers (9) showed that it was effective in enhancing fracture healing in a model of surgical fixed tibial diaphyseal fractures. However, in a recent randomized 
controlled study, this drug had no effect on healing of wrist fractures (10).

To our knowledge, no other case of sellar reossification associated with strontium ranelate use has been reported. We speculate if strontium ranelate may have affected bone mineralization at the sellar floor.

However, we cannot rule out the possibility that this association could simply be a coincidence, in a patient where reoperation was delayed 2 years from the initial surgery. Bone regrowth can happen after surgery, particularly if reoperation is postponed for a significant period of time.

In addition, we speculate if the hemorrhagic complication that this patient suffered was due to hemorrhage into the surgical field, an extrasellar hemorrhage due to lesion of intradural vessels, or direct injury to the internal carotid artery.

Injury to the internal carotid artery is a major complication associated with transsphenoidal surgery. Reported incidence ranges from 0.4 to $3.8 \%$ and is higher in endoscopic series compared to microscopic series (11). It has been associated with significant morbidity and mortality. Previous surgery, as is the case of this patient, with distorted anatomy constitutes a risk factor. Additional risk factors include adherence of the lesion to the internal carotid artery, previous radiotherapy and prior bromocriptine therapy.

We conjecture if the reossification of the sellar floor associated with the use of strontium ranelate might have directly affected the internal carotid arteries, and traction during operative manipulation causing its damage with subsequent severe hemorrhage.

In conclusion, sellar reossification should always be taken into account when considering repeated transsphenoidal surgery. Detailed preoperative evaluation of bony structures by computed tomography ought to be performed in all cases of reoperation. It remains unclear whether strontium ranelate could have affected sellar ossification. We strongly recommend that indications for prescribing this drug should be carefully followed.

\section{Declaration of interest}

The authors declare that there is no conflict of interest that could be perceived as prejudicing the impartiality of the research reported.

\section{Funding}

This research did not receive any specific grant from any funding agency in the public, commercial or not-for-profit sector.

\section{Patient consent}

No consent form was obtained because the patient is deceased.

\section{Author contribution statement}

All co-authors listed contributed substantially to the preparation of this manuscript. The authors would like to thank Dr Nicolás Sgarbi for his technical assistance with images.

\section{References}

1 Spaziante R, de Divitiis E, Cappabianca P \& Zona G 2005 Repair of the sella turcica after transsphenoidal surgery. In Schmidek and Sweet's Operative Neurosurgical Techniques. Indications, Methods and Results, pp 390-408. Eds H Schmidek \& D Roberts. Philadelphia: Saunders.

2 Bonnelye E, Chabadel A, Saltel F \& Jurdic P 2008 Dual effect of strontium ranelate: stimulation of osteoblast differentiation and inhibition of osteoclast formation and resorption in vitro. Bone $\mathbf{4 2}$ 129-138. (doi:10.1016/j.bone.2007.08.043)

3 Zona G \& Spaziante R 2007 Suboptimal sphenoid and sellar exposure. Neurosurgery 60 E582. (doi:10.1227/01.NEU.0000261577.25603.DE)

4 Yahia-Cherif M, Delpierre I, Hassid S \& De Witte O 2016 Bony regeneration of the sella after transsphenoidal pituitary surgery. World Neurosurgery 88 497-502. (doi:10.1016/j.wneu.2015.10.073)

5 Farlay D, Boivin G, Panczer G, Lalande A \& Meunier PJ 2005 Long-term strontium ranelate administration in monkeys preserves characteristics of bone mineral crystals and degree of mineralization of bone. Journal of Bone and Mineral Research 20 1569-1578. (doi:10.1359/JBMR.050405)

6 Habermann B, Kafchitsas K, Olender G, Augat P \& Kurth A 2010 Strontium ranelate enhances callus strength more than PTH 1-34 in an osteoporotic rat model of fracture healing. Calcified Tissue International 86 82-89. (doi:10.1007/s00223-009-9317-8)

7 Arlot ME, Jiang Y, Genant HK, Zhao J, Burt-Pichat B, Roux J-P, Delmas PD \& Meunier PJ 2008 Histomorphometric and microCT analysis of bone biopsies from postmenopausal osteoporotic women treated with strontium ranelate. Journal of Bone and Mineral Research 23 215-222. (doi:10.1359/jbmr.071012)

8 Tarantino U, Celi M, Saturnino L, Scialdoni A \& Cerocchi I 2010 Strontium ranelate and bone healing: report of two cases. Clinical Cases in Mineral and Bone Metabolism 7 65-68.

9 Aslam MZ, Khan MA, Chinoy MA, Jillani SAA, Sultan SA \& Ahmed SK 2014 Significance of strontium ranelate in healing of surgically fixed tibial diapyseal fractures treated with strontium ranelate vs placebo; a randomised double blind controlled trial. Journal of the Pakistan Medical Association 64 S123-S126.

10 Scaglione M, Fabbri L, Casella F \& Guido G 2016 Strontium ranelate as an adjuvant for fracture healing: clinical, radiological, and ultrasound findings in a randomized controlled study on wrist fractures. Osteoporosis International 27 211-218. (doi:10.1007/s00198-015-3266-z)

11 Ammirati M, Wei L \& Ciric I 2013 Short-term outcome of endoscopic versus microscopic pituitary adenoma surgery: a systematic review and meta-analysis. Journal of Neurology, Neurosurgery, and Psychiatry $\mathbf{8 4}$ 843-849. (doi:10.1136/jnnp-2012-303194)

Received in final form 14 May 2017

Accepted 6 June 2017 